Instead of a single real-valued solution $x(t)$ of (1), choose for (1) two solutions, $x=x(t)$ and $x=y(t)$, which are real-valued and linearly independent. In view of the superposition principle, the (complex-valued) function

$$
z(t)=x(t)+i y(t)
$$

is a solution of the (real) differential equation (1). But

$$
z(t) \neq 0 \text { and } z^{\prime}(t) \neq 0
$$

now hold on $I$ when $I$ is the entire half-line under consideration $\left(t_{0} \leqq t<\infty\right)$. In fact, since $x=x(t)$ and $x=y(t)$ are linearly independent solutions of (1), their Wronskian $x y^{\prime}-y x^{\prime}$ is a non-vanishing constant; hence neither $x$ and $y$ nor $x^{\prime}$ and $y^{\prime}$ can vanish at the same $t$, and so it is clear from (16) that both inequalities (17) are satisfied at every $t$.

Owing to the first of the inequalities (17), the logarithmic derivative

$$
m(t)=z^{\prime}(t) / z(t)
$$

exists on the entire half-line $I$. On the other hand, the second of the inequalities (17) assures that (13) defines the function $n(t)$ on the entire half-line $I$ if $m(t)$ is defined by (18).

Finally, since $x=z(t)$ is a solution of (1), Riccati's deduction of (12) from (11) and the case $x=x(t)$ of (1) remains unaltered if (11) and (1) are replaced by (17) and the case $x=z(t)$ of (1) respectively. Since the transition from (12) to (15) had nothing to do with the reality of $m(t)$, the proof is now complete.

\title{
ON THE ROTATION OF AN INCOMPRESSIBLE CONTINUOUS MEDIUM IN PLANE MOTION*
}

\section{BY WALTER NOLL (Carnegie Institute of Technology)}

We consider a plane motion of an incompressible medium with homogeneous density $\rho=$ const. We assume that the plane region occupied by the medium is of the following type: at each instant it is bounded by a finite number of smooth curves. If it is finite all but one of the closed boundary curves move rigidly. If it extends to infinity all finite closed boundary curves move rigidly. We label this motion by I. Now we consider another motion, labeled by II, which is obtained from I by superposing a uniform rotation with angular velocity $\omega=$ const. about a fixed axis perpendicular to the plane of the motion.

It will be shown that motion II is dynamically possible whenever I is, and that the stresses in II differ from those in I only by a scalar stress given explicitly in (10) below.

G. I. Taylor [1] proved this theorem for the special case of the irrotational motion of an inviscid fluid caused by rigid cylindrical bodies moving in an infinite container. It has been shown recently that Taylor's result continues to hold for linear viscous fluids [2] and for a special type of non-linear viscous fluids [4]. The main purpose of this paper is to point out that Taylor's theorem remains valid for any material whatsoever (it may even be an anisotropic solid). The essential assumptions are that the medium be incompressible and that the motion be plane.

${ }^{*}$ Received August 17, 1956. Written under Contract Nonr 228(09), Office of Naval Research. 
A motion with velocity $\mathbf{v}$ is dynamically possible if there is a stress tensor $S$ such that the continuity equation, the equation of motion, and the constitutive equation of the material are satisfied with $\nabla$ and $S$. The first two of these equations are in our case

$$
\begin{gathered}
\operatorname{div} \nabla=0 \\
\operatorname{div} S+\rho f=\rho \forall,
\end{gathered}
$$

where $f$ is the density of the extraneous forces. In general, the constitutive equation will serve to determine the stress $S$ by the motion (and possibly certain initial data). However, in an incompressible medium the motion determines the stress only up to a scalar stress $p I(I=$ unit tensor) so that only the extra stress $T=S+p I$ is completely determined. It follows from the principle of isotropy of space (see [3], p. 19) that the constitutive equation is not altered by the superposition of any rigid rotation.

We describe motion II in a frame of reference which rotates with the superposed rotation. Then both I and II will have the same velocity field $\nabla_{I}=\nabla_{I I}=\nabla$. Therefore, by the principle just mentioned, the extra stresses must be the same for both motions:

$$
T_{\mathrm{I}}=p_{\mathrm{I}} I+S_{\mathrm{I}}=p_{\mathrm{II}} I+S_{\mathrm{II}} \text {. }
$$

The equation of continuity (1) is the same for both motions. For a plane motion, (1) implies the existence of a stream function $\psi$ such that

$$
\mathbf{v}=-\operatorname{curl}(\mathbf{e} \psi)
$$

where $\mathbf{e}$ is a unit vector perpendicular to the plane of motion. The assumptions on the region mentioned in the beginning ensure that $\psi$ is single valued. The equation of motion (2) for I is

$$
\operatorname{div} S_{\mathrm{I}}+\rho \mathbf{f}=\rho \dot{v}
$$

In the rotating frame, however, we must add to $f$ a field $g$ which represents the centrifugal and Coriolis forces, so that we get

$$
\operatorname{div} S_{\mathrm{II}}+\rho(\mathbf{f}+\dot{\mathrm{g}})=\rho \dot{t}
$$

(It is understood that the extraneous forces $f$ are to be rotated also). It follows from (3), (5), and (6) that motion II will be dynamically possible if and only if $p_{\text {II }}$ can be. chosen in such a way that

$$
\rho \mathrm{g}=\operatorname{grad}\left(p_{\mathrm{II}}-p_{\mathrm{I}}\right)
$$

holds, i. e., if and only if $\mathbf{g}$ has a single valued potential. This is not the case for a general three-dimensional motion. But for a plane motion an easy analysis (see [1], p. 101) shows that

$$
\mathbf{g}=\operatorname{grad}\left(2 \omega \psi+\frac{1}{2} \omega^{2} r^{2}\right)
$$

where $r$ is the distance from the axis of rotation. It follows from (8), (7) and (3) that the pressure $p_{\text {II }}$ and the stress $S_{\text {II }}$ of motion II are given by

$$
\begin{gathered}
p_{\mathrm{II}}=p_{\mathrm{I}}+\rho\left(2 \omega \psi+\frac{1}{2} \omega^{2} r^{2}\right), \\
S_{\mathrm{II}}=S_{\mathrm{I}}-\rho\left(2 \omega \psi+\frac{1}{2} \omega^{2} r^{2}\right) I .
\end{gathered}
$$

From (10), the forces exerted on the boundaries in motion II can be obtained in the same way as was done by Taylor [1]. 
REFERENCES

1. G. I. Taylor, Proc. Roy. Soc. A93, 99-113 (1916)

2. W. R. Dean, Quart. J. of Mech. Appl. Math. 7, 257-262 (1954)

3. W. Noll, J. Ratl. Mech. and Anal. 4, 3-81 (1955)

4. M. K. Jain, Z. angew. Math. u. Mech. 35, 379-381 (1955) 